\title{
TEATRO MÍNIMO: \\ ¿NECESIDAD DE FORMA O FORMA DE NECESIDAD?
}

MINIMUM THEATER: NEED OF FORM OR FORM OF NEED?

\author{
Antonio César MORÓN \\ Universidad de Granada \\ acemores@ugr.es
}

\begin{abstract}
Resumen: Desde hace unos años el teatro breve se ha convertido en un fenómeno empresarial con unas características particulares en las que se establece una relación de consumo mucho más cercana, espontánea e informal con un tipo de espectador que generalmente huye de los montajes y los espacios tradicionales del teatro. Este artículo da cuenta del denominado Teatro Mínimo, bajo el que se engloban un grupo de actores, directores y dramaturgos de Sevilla que, durante las temporadas que van del año 2012 al año 2014, desarrollaron un proyecto teatral innovador y muy comprometido con las circunstancias socioeconómicas y políticas de los años clave de la crisis global en España.
\end{abstract}

Palabras clave: Teatro Mínimo. Sevilla. Crisis. Underground. Laboratorio.

\begin{abstract}
Short Theater has become a drama enterprise phenomenon with noteworthy characteristics. The relationships of consumption are much more close, spontaneous and informal. It attracts a type of spectator who usually shuns traditional spaces. This article gives account of the socalled Minimum Theatre, which encompasses a group of actors, directors and playwrights in Seville, between 2012 and 2014. They developed an innovative drama project and were very committed to delve into the socioeconomic and political circumstances of the main years of the last largescale crisis in Spain.
\end{abstract}

Key Words: Minimun Theater. Seville. Crisis. Underground. Laboratory. 


\section{INTRODUCCIÓN: EL PROYECTO TEATRO MÍNIMO DE SEVILLA}

\subsection{Contexto de producción del Teatro Mínimo}

Durante las temporadas 2012, 2013 y 2014 se desarrolló en la ciudad de Sevilla un proyecto teatral denominado Teatro Mínimo, en el que participó un nutrido grupo de actores, directores y dramaturgos, además de gente de la pequeña y mediana empresa de Sevilla. En el año 2015, la Consejería de Cultura de la Junta de Andalucía, a través del Centro de Documentación de las Artes Escénicas de Andalucía (CDAEA), recoge en un volumen publicado en formato electrónico una selección de diez piezas. Son textos que pertenecen concretamente a nueve autores; si bien conviene recordar que fueron exactamente cuarenta y tres quienes participaron en esta experiencia artística durante su primera temporada (Berger y otros, 2015) ${ }^{1}$. El Centro de Documentación de las Artes Escénicas de Andalucía viene publicando, de manera fehaciente, obras de teatro breve desde hace más de diez años, tras la potenciación, sin duda, que le otorgó a este género el actual director de la Escuela Superior de Arte Dramático de Sevilla, Alfonso Zurro, quien cultivó esta modalidad dramática desde los años ochenta. Como apunta Gutiérrez Carbajo:

en el teatro, la brevedad, aparte de su indudable carga conceptual, es uno de los rasgos más susceptibles de verificabilidad empirica [de manera que] algo es breve o corto [...] según los términos de mensurabilidad que establezcamos y las circunstancias pragmáticas que contextualicen la medida (Gutiérrez Carbajo, 2013: 19).

\footnotetext{
${ }^{1}$ Tales autores fueron: Alex Peña, Andrés Blanco, Antonio Estrada, Antonio Quiles, Arantxa Sanchís, Aïda Ballmann, Beatriz Cotobal, Belén Cobos, Carmen Corchs, Clara Romero, Cristina Royo, Daniela Morzolo, David Arnaiz, David Montero, Estrella Corrientes, Eva Gallego, Javier Berger, Joaquín D’Holdan, Jorge Dubarry, Jorge Naranjo, Joserra Leza, José F. Ortuño, José Luis de Blas, Juan Rivadeneyra, Laura Alvea, Laura de Arco, Mari Paz Sayago, Marta Pajuelo, María José Castañeda, Mercedes Bernal, Migue López, Pablo Baena, Paco Gámez, Paco Luna, Paqui Montoya, Pepa Solís, Pilar Herranz, Rocío Galán, Sergio Rodríguez, Silvia Rey, Tomás Afán, Verónica Moreno y Verónica Roiz.
} 
Por otro lado, recogiendo las ideas de Juan Mayorga, desde el prólogo de sus piezas breves insertas en su libro Teatro para minutos, cada uno de esos textos "quiere ser leído no como esbozo o boceto de un texto más amplio, y mucho menos como los restos de un largo texto fallido [sino] como una obra completa" (Mayorga, 2001: 7). Brevedad y sentido autónomo y completo del texto son dos conceptos que caminan conjuntamente a la hora de la interpretación, tanto desde la crítica literaria como desde su perspectiva de montaje ${ }^{2}$.

En estas primeras décadas del nuevo siglo, invadidos de una etapa postmoderna en la que el discurso filosófico o artístico aparece ampliamente fragmentado después de la crisis de los estructuralismos procedente del siglo anterior, el sistema de la cultura aparece mucho más enfocado hacia la apariencia de lo breve que hacia la rotundidad del personaje o de la historia. Tanto formatos como educación teatral del público permiten fomentar un tipo de historia que se cuenta frecuentemente con dos actores. Y lo normal es enfrentarse a un guion en el que se trasciende la realidad desde un nivel pseudo costumbrista.

\section{ACERCAMIENTO INICIAL A LAS TESIS DE TEATRO MÍNIMO A TRAVÉS DE SUS PROTAGONISTAS}

Teniendo en cuenta estas consideraciones previas que van a perfilar el discurso dramático que se analizará a lo largo de este artículo, centraré el estudio en las circunstancias socioculturales y económicas que subyacen en una expresión de este tipo, investigando acerca de quiénes son los impulsores y participantes en dicha experiencia (que supuso el inicio de un modo alternativo de espectáculo teatral), así como el grado de implicación tanto de la institución pública como de la empresa privada. Dado que lo más oportuno para conocer — desde un punto de vista empírico- una experiencia sociocultural y económica de este calado es acudir a sus fuentes más directas, he desarrollado un formulario de entrevistas con tres de sus participantes más destacados en cuanto a trayectoria teatral,

${ }^{2} \mathrm{Cf}$. a este respecto el volumen de actas del XX Seminario Internacional del Centro de Semiótica Literaria, Teatral y Nuevas Tecnologías, publicado por su director el profesor José Romera Castillo (2015), El teatro breve en los inicios del siglo XXI. 
que, además, formaron parte del núcleo primigenio de este proyecto. El formulario aludido fue enviado a cada uno de estos participantes a través de correo electrónico y devuelto por ellos mismos una vez completado.

Se podrá apreciar un lenguaje subjetivo en las respuestas de los entrevistados, mediante el cual se recogerán de forma íntima y espontánea unas reflexiones que iré intercalando en diversos apartados de carácter más objetivo y lenguaje más teórico, con el fin de confirmar algunas de las conclusiones a las que llegan dichos participantes. Los autores entrevistados han sido Javier Berger, José Luis de Blas y Jorge Dubarry. Por otro lado, es necesario destacar que otra parte de la investigación se ha llevado cabo a través de distintas plataformas - fundamentalmente blogs particularesa las que de forma habitual acceden los autores para publicar sus ideas estéticas y temáticas acerca de este fenómeno.

A la pregunta múltiple que formaba parte del primer bloque del cuestionario: "¿Procedes del mundo del teatro: fuiste actor, director, productor, etc... antes que escritor de teatro? ¿Cuándo empezaste a escribir teatro? ¿Por qué te decidiste a escribir teatro? ¿En qué salas has estrenado?", las respuestas de cada uno de ellos pueden servir, sin duda, de carta de presentación; lo cual - como ya ha sido aludido en el párrafo anterior - supondrá una contextualización importante de las palabras y reflexiones que se irán poniendo en común a lo largo del desarrollo teórico de este artículo:

He sido actor, autor, productor, director, técnico ocasional... En fin, teatrero. / Empecé a escribir teatro en el grupo de teatro de la Facultad de Ciencias en Granada. Cuando en 1997 gané el premio Romero Esteo, pensé que podría convertirse en un oficio. / Me dediqué a escribir teatro para poder pasar los veranos en Marbella (soy de alli) sin aburrirme, asi estaba cerca de la escena, aunque fuera de una forma virtual, y me evadía de la Costa del Sol y sus locuras inmobiliarias y familiares. / En el Teatro Central (Sevilla), en la Sala Cero (Sevilla), en el Gran Teatro de Córdoba... Los estrenos siempre son cerca de donde uno vive (Berger, 2015b: s. p.).

Empecé en 2005, por una casualidad. Yo escribia narrativa y guión de cine. Un actor me pidió un texto para montarlo con su grupo de teatro al que le daba clases el director Antonio Reina en la escuela 
Viento Sur. Luego hice más cosas con este director (De Blas, 2015: s. p.).

Empecé a hacer teatro a través del mundo de la interpretación en mis últimos años de instituto, allí conecté con dos compañeros e iniciamos un grupo literario que bautizamos con el nombre de "Plumas soñadoras". Nos reuníamos para fumar en pipa e intercambiar nuestros escritos poéticos, pequeños relatos y obras de teatro. Escribiamos, dirigíamos y representábamos nuestras propias obras ... poco a poco, y casi sin darme cuenta, fui asumiendo el papel de autor-director. Esto me llevó a estudiar dirección y escenografía en el antiguo Instituto del Teatro, donde me formé, de manera paralela, con algunas de las plumas contemporáneas del momento... Michel Azama, Antonio Onetti, April de Angelis, Sarah Kane, etc. / Mis obras se han estrenado en Sala Cero, Sala Imperdible, La fundición, Gran Teatro de Córdoba, Festival Grec de Barcelona, Sala Triangulo... (Dubarry, 2015: s. p.).

Es interesante observar cómo cada uno de ellos asegura haber llegado al mundo de la escritura dramática a través de tres vías principales: bien la casualidad, procedente del ofrecimiento inesperado de alguna compañía o director conocido; bien la evasión; bien la búsqueda de un premio literario.

Javier Berger, quien — como se anunció anteriormente — aparte de ser uno de los dramaturgos más implicados en este proyecto es uno de sus más férreos impulsores, nos habla acerca de los inicios de lo que él mismo denomina el Mínimo o lo Mínimo, "A modo de prólogo":

Tres tipos: Sergio Rodríguez, Jorge Dubarry y yo mismo: Javier Berger junto a los dinamizadores de Un Gato en Bicicleta: Raquel Eidem y Jesús Barrera somos, de momento, el núcleo duro de Teatro Mínimo (o el Mínimo, como se le conoce). Fue Sergio Rodríguez, el que, junto a Jesús Barrera, decidió lanzarse a esta locura. La idea la robaría de algún sitio, como hacía Shakespeare los días de lluvia (Berger y otros, 2015: 4).

Se cuenta en total con tres autores y dos empresarios que cedieron 
el espacio teatral alternativo en el que se desarrollaría la mayoría de los montajes programados. El dramaturgo José Luis de Blas, en el segundo prólogo de este libro, habla del bar La Traviesa, como otro de los espacios utilizados en las diversas funciones que se llevaron a cabo. Convendría analizar como primer hecho destacable la huida de estos dramaturgos hacia espacios escénicos no convencionales: una librería y un bar. Ahora bien, más allá de esto, lo que sería pertinente plantearse es el porqué de esa huida o rechazo a lo convencional: ¿cuál es el fin del mismo? Es cierto que se pueden establecer varios tipos de argumentos para responder a esta última pregunta. Pero hallamos, en primer lugar, una razón económica más que evidente en cuanto a la intención de llevar a cabo esta huida.

\section{LA SITUACIÓN DE LA INDUSTRIA TEATRAL EN ANDALUCÍA Y LA COMPLEJA RELACIÓN CON LOS PROGRAMAS DE APOYO INSTITUCIONAL POR PARTE DE TEATRO MÍNIMO}

\subsection{Teatro y apoyo institucional desde la Junta de Andalucía}

Cuando aparece la crisis que, a partir de la caída de importantes bancos estadounidenses en 2008 sacude la economía mundial y, por supuesto, la española, encontramos en Andalucía un tejido profesional destrozado, sin posibilidad alguna de absorber a la cantidad de actores egresados de la ESAD de Sevilla ${ }^{3}$. Conviene recordar con la investigadora María Jesús Bajo Martínez, que la industria teatral en Andalucía había sido muy compleja de reconstruir tras la desaparición paulatina de la misma tras la Guerra Civil, y cómo en los años noventa, tras un ambicioso plan de rehabilitación de teatros públicos, "iniciado por los ministerios de Cultura y Obras Públicas, y continuado por las consejerías homónimas, tras haber asumido la Junta de Andalucía las competencias correspondientes" (Bajo Martínez, 1996: 34), se habían efectuado hasta el momento hasta veintinueve rehabilitaciones sobre distintos edificios teatrales diseminados por todo el mapa de la Comunidad. De tal manera que, en el año 1996, la

\footnotetext{
${ }^{3}$ A la que habría que sumar también los alumnos egresados en la Comunidad de la ESAD de Málaga y de la de Córdoba, aparte de las decenas de escuelas más o menos oficiales que proliferan en cada una de las provincias andaluzas.
} 
investigadora citada concluye diciendo que:

Andalucía vive en la actualidad un buen momento teatral, no exento de dificultades de distribución y penurias económicas, de compañias que nacen y que mueren precipitadamente. Pero el interés por este arte, sin duda, es grande, como lo demuestran los numerosos autores y directores, y las más de trescientas compañías existentes, de las que cerca del centenar son profesionales. Todos los géneros y estilos tienen su público (Bajo Martínez, 1996: 34).

Siguiendo dentro de esta senda de optimismo, aunque estableciendo algún que otro reparo, resulta interesante destacar la reflexión del investigador de la Universidad de Sevilla, Ramón Espejo Romero, en un prólogo de obras conjuntas de teatro breve de autores andaluces, publicado en el año 2005. El investigador incide en el buen momento que atraviesa el teatro andaluz, si bien "la escritura dramática no ha sabido beneficiarse de dicho interés, quizás por falta de un marco adecuado en el que desarrollarse o de fomento por parte de los poderes públicos" (Espejo Romero, 2005: 5). Unas líneas más abajo, matiza esta afirmación inicial subrayando que "los autores que deciden quedarse en y escribir desde Andalucía cuentan con mayores facilidades para hacerlo" (Espejo Romero, 2005: 6), llegando a constatar que, en lo referente al teatro breve, se está viviendo una de sus épocas doradas dentro de la Comunidad Andaluza. Esta gradación reflexiva en la que se pasa de la falta de fomento por parte de los poderes públicos a las facilidades para desarrollar la escritura en Andalucía, indica que a medida que avanza el nuevo milenio nos encontramos con un momento complejo, en el que la institución apoya a los dramaturgos. Pero se tiene la sensación de que, de manera paulatina, se va revirtiendo aquel optimismo del año 1996 que veíamos reflejado en el artículo de la investigadora María Jesús Bajo.

La publicación de textos recopilatorios de teatro breve de autores andaluces - como el mismo en el que Espejo Romero inscribe su prólogo - u otros más recientes — como el de Día mundial del teatro. Sevilla 2010. (Sevilla, Junta de Andalucía, 2010) o el de 60 obras de 1 minuto de 60 autores dramáticos andaluces. (Sevilla, Junta de Andalucía, 2006) - , evidencian el apoyo que desde la institución se le ha conferido a este género dramático. El montaje dramático institucional de una obra 
resulta mucho más costoso (hablando en términos económicos) que la publicación de un libro. Aunque es de rigor destacar que, en el año 2004, Alfonso Zurro dirige para el Centro Andaluz de Teatro el espectáculo Los siete pecados capitales, en el que incluye obras breves de siete autores andaluces (Espejo Romero, 2005).

La conclusión que surge de todo lo comentado en párrafos anteriores es que la institución no puede dar salida en su totalidad a un panorama tan rico y tan vasto como el teatro escrito en Andalucía. Tampoco tiene posibilidades para amparar a todos los egresados de las distintas Escuelas de Arte Dramático de la Comunidad. Como problema añadido, nos encontramos con la realidad de que en Andalucía no existen entidades privadas con la fuerza suficiente para albergar a las múltiples promociones que durante años han estado egresando de estas escuelas. Así, esos actores que dejan de ser llamados para llevar a cabo producciones teatrales se ven abocados a convertirse ellos mismos en productores. No hay que olvidar, por otro lado, que un espacio dramático convencional conlleva un coste de alquiler que solo las grandes productoras privadas —o aquellas que están subvencionadas - pueden permitirse. Así, dentro de este panorama, el máximo ingenio se hace necesario, como se demuestra en el proyecto que llevó a cabo Alfonso Zurro a través de una colaboración entre la ESAD de Sevilla y la Consejería de Cultura de la Junta de Andalucía en el año 2010 para celebrar el Día Mundial del teatro, desarrollando en cada una de las habitaciones de un hotel obras de teatro breve en torno a tres minutos de duración (Zurro, 2010). Esta es, sin duda, la influencia más cercana que tuvieron los autores del Teatro Mínimo, muchos de los cuales habían participado en este proyecto de Zurro que, en el año 2006, como director de la ESAD de Sevilla, expuso en breves líneas aquello que constituye la compleja y productiva relación que desde el inicio del nuevo milenio se ha venido estableciendo entre la institución política y administrativa de la Junta de Andalucía y los diferentes agentes generadores del hecho escénico:

El teatro andaluz, en su conjunto, tiene un vigor creativo al que hay que darle salida para que llegue en las mejores condiciones al público, y necesita de buenas dosis de imaginación para activarlo desde sus poliédricas posibilidades. I El futuro del teatro no solo radica en recuperar o construir teatros y en apoyar empresas de 
producción, tan importantes como ello es potenciar la escritura dramática. Conseguir que un teatro vivo pertenezca a una sociedad porque es escrito para ella; y asi ayude a comprendernos, refleje nuestras paradojas, y sobre todo los grandes interrogantes humanos desde nuestro propio tiempo (Zurro, 2006).

\subsection{La compleja relación de los autores de Teatro Mínimo con la institución}

Los autores de lo Mínimo en Sevilla establecen una relación compleja con la institución, que se podría catalogar como de ida y vuelta o de salida y entrada. Esta relación está condicionada por una circunstancia fundamental: el núcleo que saca este proyecto adelante son, además de autores, actores y/o directores. La institución no financia el montaje de sus textos, pero sí los acaba recogiendo y publicando en un volumen. De modo que se podría concluir diciendo que salen de la institución a causa de la imposibilidad misma de ser amparados por ella en cuanto al montaje de estas obras, pero siguen vinculados a muchos de los proyectos institucionales que se llevan a cabo: desde la participación en otros montajes a la impartición de talleres o a la publicación de algunos de sus textos de manera conjunta. Aunque sería demasiado naif pensar que solo ese supuesto abandono y la necesidad de seguir creando los lleva a recorrer sus pasos en este sentido. Considero, pues, que su independencia está relacionada también con la búsqueda de una libertad absoluta para ejercer una crítica social, a partir de un lenguaje y unas situaciones que difícilmente aceptaría la institución política. Porque si hay algo que define la obra de estos dramaturgos es la idea de ser políticamente incorrectos. A la idea de independencia desde un punto de vista político, para exponer lenguajes y temáticas que, quizás, costara trabajo que la institución subvencionara (de esto se hablará con más detalle en párrafos posteriores), hay que sumar la idea de independencia estética, que posibilita un marco de investigación dramatúrgica y escénica a través de este formato. Los autores que participaron de esta experiencia tenían muy clara esta última perspectiva. En este sentido, conviene analizar con detalle las respuestas que efectuaron los tres autores encuestados a la pregunta "¿Por qué Teatro Mínimo: por necesidad o por gusto por ese formato concreto?”: 
El fenómeno se ha consolidado como necesidad, pero no es ninguna solución a la precariedad de la profesión. Pues se establecen baremos y condiciones aún peores $y$, en ocasiones, cimentan un amateurismo preocupante. Es un buen lugar para experimentar, probar, pero no para quedarte ahi y tratar de vivir de ello (Berger, 2015b: s. p.).

Para muchos el Teatro Mínimo ha sido como un clavo ardiendo, la necesidad ha empujado a muchos a hacer cualquier cosa antes de permanecer inactivos. Por otra parte, yo no creo en un formato concreto. Cada pieza es libre de escoger su extensión, incluso de seguir creciendo o detenerse, quizá tras un tiempo, convertirse en otra cosa. El teatro breve es al teatro de duración habitual lo que un cuento a una novela, y ha de crear sus propios mecanismos. Algunos de mis textos breves sé que tienen la duración exacta, están "cerrados" de alguna manera, mientras que hay otros en los que intuyo huecos, o caminos donde seguir trabajando (De Blas, 2015: s. p.).

Primero por necesidad de seguir creando y estar en activo, luego te das cuenta de que ha servido para abrir una puerta de investigación a lo más profundo, al menos para mí (Dubarry, 2015: s. p.).

Se evidencia cómo, si en un primer momento este formato surge por pura necesidad, hay posteriormente un interés por comprenderlo como un espacio de creación, al menos durante el tiempo que el proyecto esté dando frutos (aunque mínimos) económicos y de público. La imagen del temor a la inactividad que destaca José Luis de Blas en su respuesta deja patente la consideración de lo Mínimo como una especie de salvavidas para muchos de los teatreros implicados en el proyecto.

\section{LO MÍNIMO ENTRE EL CONTEXTO TEÓRICO DEL TEATRO DE CORTA EXTENSIÓN Y LA ÓPTICA DE SUS AUTORES}

\subsection{Aproximación al contexto teórico del teatro breve}


Definir la finalidad de lo Mínimo dentro de un argumento concreto y único, resulta complicado, como se puede observar al hilo de toda la exposición precedente. Ahora bien, intentando responder con mayor detalle a esta pregunta formulada unos párrafos más arriba — ¿cuál es el fin de lo Mínimo? - convendría centrarse en primer lugar en definir qué es el Teatro Mínimo, situándolo en un contexto determinado, dado que teatro de corta extensión ha existido siempre, desde que se escribe teatro como tal. Está claro que el teatro breve no siempre ha sido considerado de la misma manera ni con los mismos fines. La experiencia del Teatro Mínimo está muy alejada en sus pretensiones de las obras cortas que plantearan autores como los de la denominada "Otra Generación del 27"4. Existe un estudio fundamental de Eduardo Pérez-Rasilla, en donde el investigador realiza la siguiente reflexión acerca del teatro breve de estos:

Jardiel, Mihura o Neville escribieron obras breves destinadas a la publicación en revistas literarias o de humor, sin pensar en su escenificación, porque se trataba de un ejercicio estilístico ligero, redactado sin demasiadas pretensiones, destinado a cubrir las colaboraciones que les solicitaban. No faltan tampoco piezas que constituyen borradores de comedias extensas, ni tentativas que llegaron de forma autónoma a los escenarios de los teatros experimentales, universitarios o de cámara (Pérez-Rasilla, 2008: 1153).

En contra, pues, de la consideración que un dramaturgo como Juan Mayorga tiene acerca del teatro de corta extensión - consideración a la que se ha aludido en la introducción de este artículo-, aquellos dramaturgos del 27 consideraban este teatro como una especie de género menor, incluso un borrador de sus obras extensas, que publicaban a modo de colaboración ante la solicitud de las diversas revistas literarias de la época. Si he traído esta cita a colación es porque me permite destacar una tendencia en la consideración de este tipo de teatro, que ha sido la que ha imperado a lo largo del tiempo y que muy bien resume Pérez-Rasilla en el sintagma "ejercicio estilístico ligero". Es curioso a este respecto detenerse

\footnotetext{
${ }^{4}$ Sin olvidar a los Quintero, aunque más alejados que la Otra Generación del 27, más presentes en la cultura popular andaluza y en su memoria colectiva.
} 
a considerar que entre las cuarenta entradas caracterizando al teatro que recoge el investigador francés Patrice Pavis en su Diccionario del Teatro, no encontramos en ningún caso la etiqueta Teatro breve o alguna parecida. Esto solo puede significar una cosa: que para Pavis la idea de brevedad no establece ninguna tipología en sí misma, al margen de que pueda estar asociada a diferentes tipologías, como podemos observar que hace al definir la palabra entremés como "obra cómica corta" (Pavis, 1998: 161). Sí encuentra, sin embargo, Pavis una etiqueta para caracterizar este tipo de teatro: teatro alternativo. Aunque no destaca dentro del mismo la brevedad como ingrediente fundamental:

La alternativa al teatro comercial y al teatro público subvencionado es, con todas sus dificultades, la que ofrecen o bien el teatro experimental o un tercer teatro que propone una programación, un estilo y un modo de funcionamiento completamente originales. Paradójicamente, la modestia de los medios permite probar formas nuevas con mayor iniciativa, y en una total independencia tanto económica como estética (Pavis, 1998: 437).

Me interesa la relación que establece Pavis entre "modestia de medios", "mayor iniciativa" e "independencia" económica y estética. Aunque él lo destaque como algo paradójico, es cierto que cuando un estilo teatral nace o se desarrolla al margen de los medios económicos, finalmente deja de deberle nada a esos medios económicos que en potencia podrían financiarlo. Aunque también es cierto que la falta absoluta de medios origina en muchas ocasiones la destrucción total de lo escénico.

\subsection{Aproximación a la originalidad de Teatro Mínimo desde la óptica de sus propios autores}

En este sentido, y siguiendo con la reflexión crítica del dramaturgo Javier Berger, vale la pena rescatar dos de sus textos teóricos fundamentales en los que, casi a modo de manifiesto, resuelve esta difícil paradoja que oscila entre la libertad absoluta y la destrucción, llevando las propuestas de lo Mínimo hacia cuestionamientos de teoría dramática clásica del siglo XX. Ambos textos son imprescindibles para conocer de una manera profunda qué supuso esta expresión artística. El primero de ellos es el ya 
citado "A modo de prólogo", en el que, tras la inicial contextualización del nacimiento de la idea, escribe lo siguiente:

El Mínimo es un gimnasio, un laboratorio, una trinchera de creación en estos tiempos de bombardeos a la cultura. Nos movemos por el placer de la escena, del contacto directo con el público, la cercanía, la inmediatez, la risa, el buen hacer, el aplauso a lo imprescindible. // El Mínimo no es ninguna solución. La manoseada "crisis" ha reventado compañias, teatros, instituciones, productoras... El Mínimo es un canto al oficio, libre de burocracias, de chupatintas, de famositos y de imbéciles que buscan como topos el resplandor de un flash. Teatro Mínimo es Teatro. Pero no es ninguna solución. // El Mínimo permite devolver el Teatro a los autores y a los actores. Ellos son los que quieren involucrarse en este bonito show; volver a usar los baños de camerinos, bailar encima de las mesas, iluminar con tres flexos, revolver en sus armarios, ensayar en los salones. Los que nos dedicamos a este oficio, que anda en peligro de extinción, lo amamos por encima de todo. Y por ello seguimos adelante. // El Mínimo es una guerrilla, pura supervivencia. El underground se ha comprado una escalera y se pasea por librerías, por mercerías, por boutiques. El teatro nunca necesitó millones de petrodólares, $n i$ sobres sin remitente, ni caballos, ni orquesta de clarines y tambores para existir. El teatro siempre estuvo allí. El problema es que los autores, los directores, los actores, las actrices, los escenógrafos, los técnicos... se empeñan en comer todos los días. iQué fea costumbre! (Berger y otros, 2015: 4).

Dos tendencias fundamentales de la teoría teatral dirigen estas palabras: Jerzy Grotowski, en cuanto al teatro pobre; y Eugenio Barba, en cuanto a la antropología teatral. Las ideas que el texto de Berger comparte con ambas podrían especificarse en: la consideración del teatro reducido a la mínima expresión y centrado en el cuerpo del actor, eliminando todo lo que de superficial lo desvirtúa; el teatro como estado trascendental: principio, fin y guía de la propia vida del actor; y el teatro como entrenamiento, como lucha cotidiana, capaz de otorgar la verdadera libertad. Por todo ello el teatro, tal y como plantea Berger, es la única no solución posible a la crisis, el único lugar donde habitar, que ha de ser devuelto - ganado en 
lucha de trinchera - a sus verdaderos dueños, el actor y el dramaturgo, que representan en esta cita todo lo opuesto al interés comercial que pervierte el teatro. El Teatro Mínimo es concebido, así, como una especie de retiro urbano antropológico, parecido en cierto modo a los retiros promovidos por lo que el Odin Teatret de Eugenio Barba considera "teatro de fractura", y que consisten:

en el rechazo de cualquier pragmatismo miope, y da vida, con coherencia orgánica, a modelos de la propia conciencia profesional. Como "guerrilleros", sus actores viven una vida aislada, siempre a la ofensiva, vigilados por la opinión pública de los ortodoxos [...] La táctica de este "teatro de fractura" consiste en trabajar completamente aislado, lejos de los círculos artísticos oficiales, imponiéndose sin cesar tareas siempre más arduas, nunca satisfecho de los resultados, encarnizándose para superar los obstáculos de la profesión (Barba, 1997: 34-35).

La segunda reflexión del autor Javier Berger acerca del Teatro Mínimo la encontramos desarrollada con una retórica y un formato mucho más poético, escrito en verso, que el dramaturgo dio a conocer durante la presentación del libro señalado y que, más tarde, publicaría en forma de post dentro de su blog titulado "El ring de la mentira", el día 14 de octubre de 2015:

Lo mínimo

lo mínimo que se despacha

lo mínimo que se aconseja

lo mínimo que se descarta

lo mínimo que no aparenta

Teatro mínimo fue

un gimnasio,

un laboratorio,

una trinchera en tiempos de guerra.

Fue el placer del aplauso, 
la piel entre el público,

fue el teatro verdaderamente pobre.

Teatro Mínimo fue

volver a los actores,

a los autores,

a revolver en los armarios,

a iluminar con tres flexos,

a bailar en los baños,

a cantar en las aceras,

y ensayar en los salones.

Teatro Mínimo fue

un canto al oficio,

un manifiesto libre de burócratas,

de subvenciones,

de altas y bajas.

Teatro Mínimo fue

el asidero del último superviviente,

el ungerground con escalera de color,

el teatro sin bambalinas,

sin orquesta,

sin diablas,

sin sobres ni remitentes.

Teatro Mínimo fue el teatro que siempre estuvo allí.

Un nuevo intento de salvar un oficio

en peligro de extinción.

Teatro Mínimo no fue "El Teatro".

Teatro Mínimo fue nuestra declaración de amor

(Berger, 2015a: s.p.).

Es evidente que entre una y otra reflexión existen múltiples concomitancias referidas a la búsqueda de una libertad creativa absoluta, "libre de burócratas / de subvenciones", underground, al margen de 
cualquier tipo de oficialismo. Los dos versos finales resumen con ingenio la conexión emocional de esta experiencia con los propios creadores que la hicieron posible e, incluso, pública. Esta es la razón por la cual el Teatro Mínimo se aprecia como una "declaración de amor". Así, el teatro es considerado como un mecanismo a través del cual establecer la conexión comunicativa máxima con el ser humano.

Por su parte, José Luis de Blas, en el segundo prólogo del libro citado, habla así acerca de cuál es su visión de lo Mínimo como teatro, a través de su experiencia no solo como autor, sino también como espectador:

Es un Teatro "Mínimo", no tanto por la extensión de las piezas como, me da la impresión, su faceta de búsqueda de lo esencial que subyace a cualquier representación (o presentación) teatral, la búsqueda de la porción más pequeña provista de significación escénica. En este sentido, esta propuesta cobra su mayor sentido y pertinencia, marcada indefectiblemente por la necesidad de mantener viva una voz que desde las instituciones se ha querido silenciar sin descanso, ahora que prohibir y censurar se vuelve tan poco democrático y políticamente incorrecto. En este contexto, Teatro Mínimo arranca con una premisa temática que define la circunstancia social a la que debe, probablemente, su razón de ser. Las piezas, como suele ser habitual en el teatro andaluz, respiran una gran variedad de estilos y personalidades, cosa que me agrada sobremanera, y un componente de riesgo que me congracia con lo valiente y comprometido de nuestra dramaturgia (Berger y otros, 2015: 5).

Es destacable la inquietud trascendental que desarrolla José Luis de Blas en su prólogo, estableciendo el énfasis del Teatro Mínimo en una especie de búsqueda semántica de la menor unidad escénica posible cargada de significado. La visión de este autor está mucho más enfocada desde la lingüística. Cabría pensar a través de sus palabras en lo Mínimo como una unidad elemental capacitada para articular esa dramaturgia andaluza, de la cual destaca como componente fundamental el riesgo. Esto entronca de forma definitiva con la idea destacada anteriormente de huida de los espacios escénicos comunes en busca de una libertad creadora absoluta. En el fondo, de lo que nos están hablando ambos prologuistas es de la 
construcción de una nueva perspectiva para el lenguaje dramático andaluz, que habría de experimentar con los semas lingüístico-escénicos de lo Mínimo $^{5}$, que habría de separarse de manera radical de otros proyectos parecidos en el formato, pero diametralmente opuestos en su finalidad. Me estoy refiriendo al concepto relativamente nuevo de Microteatro.

\subsection{Teatro Mínimo frente a Microteatro}

En el año 2012, tras su enorme éxito de público en Madrid, abre en Sevilla esta marca comercial que apuesta por definirse, no solo temporal sino también temáticamente, como "micro-obras de entre diez y quince minutos sobre un mismo tema, representadas en espacios pequeños para menos de quince espectadores por pase y varias sesiones al día" (Microteatro, s. f.). Sin embargo, en el caso de la marca madrileña se apuesta decididamente por una finalidad económica, evidenciándolo así mismo en su eslogan comercial: "Microteatro por dinero". El Teatro Mínimo, sin embargo, deja patente en sus manifiestos un orgullo de ser "verdaderamente pobre", lo cual, siguiendo el hilo del argumento, queda de forma inmediata ligado a ser verdaderamente libre. No puede establecerse nada más opuesto al rédito económico que ese "acto de amor" al que se refieren los versos finales de la cita anterior de Berger. El Teatro Mínimo no concibe al espectador como público, sino como familia. El propio Berger presenta en nuestra entrevista las diferencias que se han venido comentando hasta este momento:

Aunque parezca sólo una diferencia de conceptos, el término "microteatro" está ligado a la marca "Microteatro por dinero" de Madrid y de sus distintas sucursales en el país y en el extranjero. Teatro Mínimo fue una propuesta más salvaje, más romántica, una postura casi de guerrilla teatral frente al desmantelamiento de todo

\footnotetext{
${ }^{5}$ Muchas de las críticas lanzadas por gran parte de la comunidad teatral hacia la gestión del antiguo Centro Andaluz de Teatro (CAT), se fundamentaban en la idea de que estaba más centrado en generar espectáculos con numeroso público, en vez de centrarse en investigar acerca de un lenguaje dramático propio, un lenguaje dramático andaluz, dirigido fundamentalmente hacia los andaluces, desde unos códigos perfectamente reconocibles. Esto ocurre con grupos andaluces de éxito internacional como La Zaranda o Atalaya; pero la Junta de Andalucía está todavía en la tarea, de construir un teatro público, con visión de futuro y basado en una investigación escénica permanente.
} 
el modelo teatral tal y como lo conocíamos. La reducción de salas, de ayudas, el cierre de compañías. Teatro Mínimo fue para una serie de profesionales un refugio, un gimnasio, un laboratorio. Nunca tuvo una visión empresarial, ningún plan de viabilidad, ni ningún anclaje económico. Fue una opción más de supervivencia. De hecho, ni hay una marca, ni una búsqueda de un nombre corporativo, ni nada similar (Berger, 2015b: s. p.).

\section{TEATRO MÍNIMO Y LA CRISIS ECONÓMICA OCCIDENTAL FOCALIZADA EN ESPAÑA}

Es curioso cómo se posiciona al Teatro Mínimo como respuesta "al desmantelamiento de todo el modelo teatral tal y como lo conocíamos". Y es que esta experiencia - ya lo hemos apuntado - no deja de estar ligada profundamente a la crisis y a los efectos de la misma, que supusieron, como dice Berger, "la reducción de salas, de ayudas, el cierre de compañías". De ahí que lo Mínimo se llegue a concebir como una supervivencia. Ahora bien, cabría matizar que no se está refiriendo el autor en ningún caso a una supervivencia económica, sino, más bien, a la posibilidad de continuar ejerciendo una pasión que se les niega como efecto de la caída económica que experimenta España desde 2008, agravada por la explosión de la burbuja inmobiliaria que sucede a partir del año 2010. Ya fue destacado al comienzo de este artículo cómo los autores dramáticos del Teatro Mínimo cobran conciencia de esta debacle, dado que uno de los sectores más afectados por la crisis ha sido, sin duda, el sector cultural y, muy especialmente, el de las artes escénicas. A la pregunta " ¿Ha afectado la crisis a tu concepción de la escritura dramática?", encontramos dos respuestas interesantes de parte de José Luis de Blas y Javier Berger:

En este sentido, sí. Para teatro breve es inviable más de 2 actores por razones de economía. Yo hasta ahora monto en situación de cooperativa. Todos sacamos lo mismo. Por eso y por las condiciones logísticas (De Blas: 2015: s. p.).

Ha afectado en la temática. Vives, lees la prensa, la precariedad se ha instalado como un modo de vida, y ya se ha aceptado que esto es 
así. ¿Recordáis cuando, no hace tanto, todos trabajábamos dados de alta? Ah, y cuando se pagaban los ensayos según convenio... ¿te acuerdas? Pues no hace tanto (Berger, 2015b: s. p.).

La respuesta de Javier Berger se carga todavía más de sarcasmo e ironía ante una pregunta de aspecto mucho más sociológico como “¿Qué es la crisis para ti?":

Un buen invento del capitalismo para seguir exprimiéndonos, generar miedo y que pueda vender la precariedad en pos de un futuro mejor (tal y como hacen todas las religiones monoteístas) (Berger, 2015b: s. p.).

El caos de la recesión inicial, el aumento de los índices macroeconómicos de déficit (como la prima de riesgo) y los recortes marcados desde Bruselas al estado español, gestionados por los gobiernos del Partido Socialista, primero, y del Partido Popular, después, suponen una pérdida en el poder adquisitivo de todos los españoles y de todas las instituciones públicas. Dentro de un escenario como este el mundo de la cultura en general y muy especialmente el de la cultura subvencionada, se ve afectado en todas sus dimensiones ${ }^{6}$. Si la reducción de medios ha influido incluso en las propias instituciones creadas por la Junta, podemos imaginar que para la profesión teatral ha sido mucho más devastadora. Los autores encuestados responden con una profunda desazón con respecto al panorama generado, cuando se les plantea la pregunta: “¿Podrías hablarme de la política de subvenciones públicas? Qué consideras y a cuáles de ellas te has acogido para publicar y/o estrenar tus textos dramáticos”:

Es complicado. Creo que deberían dar más importancia a la distribución. Andalucía dispone de una red de teatros infrautilizada. Buscar alternativas como la creación de compañias estables en teatros municipales. Se ha acabado un modelo y, de momento, no hay ningún otro. La política cultural y teatral en Andalucía es un

\footnotetext{
${ }^{6}$ Conviene recordar cómo antes de los años oficiales de la crisis, por múltiples razones aunque, sin duda alguna, también razones de índole económico, ya habían caído escuelas de formación del prestigio del Instituto del Teatro de Sevilla (Quintana, 2008).
} 
auténtico desastre. De nada sirve ayudar a la producción, si después te "comes con patatas" la función. Para la Junta o el Ministerio resulta más sencillo destinar un dinero a una serie de compañias $y$ después desentenderse que tener una verdadera política teatral, un seguimiento. Yo prefiero que me concedan cuarenta funciones a que me den una ayuda para montar un espectáculo que después no podré mostrar en ningún teatro. Montar una función no es lo más difícil del proceso, saber dónde estrenar, dónde representar, cómo poder vivir de ello. El número de funciones que hace una compañia solvente al año se ha reducido a menos de la mitad en los últimos años. No tiene sentido que tengas que ir plaza por plaza para vender tu espectáculo, dándole a los programadores culturales de los pueblos un poder absoluto y, normalmente, teniendo que plegarte a unos criterios absurdos y arbitrarios. // En fin, los teatreros tenemos ideas, nuestros politicos solo se acercan al sector cuando llaman las urnas y después "ciao". Hay que buscar alternativas a un modelo fracasado, dependiente de lo público y que ha demostrado ser un desastre (Berger, 2015b: s. p.).

Jamás he recibido subvención alguna, aunque sólo he presentado un texto, el "Doña Inés", y no conseguimos nada. De hecho, los textos que he escrito y que se han montado todos eran iniciativas privadas (De Blas, 2015: s. p.).

La fórmula de subvenciones públicas que existe no funciona, ya que, únicamente se nutren unos pocos de ellas, dejando en la cuneta a multitud de compañias que deben autogestionarse, por lo que es muy difícil poder competir después en el mercado, ya que, son las mismas compañias subvencionadas las que ocupan después los espacios públicos, dejando sin opciones a las pequeñas compañias independientes... la pescadilla que se muerde la cola. // En mi opinión, el trabajo que tienen que hacer desde las administraciones es impulsar las producciones, la promoción y difusión de obras, crear una verdadera red de teatro en la que todas las compañias tengan cabida. Abrir los espacios públicos (la mayoría están cerrados la mayor parte del año). Hacer un trabajo exhaustivo de creación de público, y sobre todo, fomentar las enseñanzas artísticas en las 
escuelas (Dubarry, 2015: s. p.).

Hay varias ideas básicas que se distribuyen a lo largo de estas tres citas. En primer lugar, se confirma la dificultad para conseguir una subvención, llevando al autor a la iniciativa privada en la mayoría de los casos. Subyace la consideración de que la mayoría de los grupos teatrales tienen dificultades para acceder a la institución. Y así, se destaca la idea de que la subvención de unas compañías deja sin opciones de estrenar a otras compañías de carácter más pequeño o independiente. Para terminar, diciendo que, en vez de subvencionar, se debería intentar desde la institución distribuir el montaje. Se señala, por otro lado, la idea de que la aproximación de los políticos al teatro suele ser esporádica y marcada fundamentalmente por el calendario electoral. Y que, para incentivar la salud del teatro, es necesario fomentar las enseñanzas artísticas en las escuelas ${ }^{7}$. La conclusión última de estos autores pone de manifiesto la idea de que el modelo de subvenciones ha fracasado y es necesario buscar una alternativa. Andalucía posee una fantástica red de teatros públicos que, a todas luces, habría que explotar mejor. Si bien es cierto que, para muchos autores y directores ocurre lo que, de un modo rotundo nos expone como una constante paradoja establecida entre subvención y producto, un teórico de la cultura como Adrian Heathfried:

Tan pronto como la dramaturgia entra en el marco institucional el dramaturgo está obligado a preguntarse: ¿qué movimientos de asimilación puede estar legitimando mi trabajo?, ¿hasta qué punto no es mi rol un instrumento de las fuerzas organizativas y

\footnotetext{
${ }^{7}$ Uno de los ejemplos que saltan a la vista de esto último es la educación en Argentina, donde desde la misma escuela existe una asignatura de teatro, con lo cual se multiplica la necesidad de profesorado para acaparar esa disciplina. Esto supone no solo la posibilidad de formar desde la infancia a los ciudadanos para que se interesen por el teatro, sino que, además, es una magnífica salida, desde la pedagogía, para gran cantidad de egresados en las escuelas de Arte Dramático. El requerimiento de este profesorado incluye una especialización fundamental de todos los futuros alumnos de las escuelas de teatro, distinguiéndose entre enseñanzas específicas para aquellos que orientan su faceta laboral a la pedagogía teatral; y enseñanzas específicas para aquellos que la orientan hacia el ejercicio técnico de la profesión como tal.
} 
tecnocráticas que están fuera de mi control?, ¿cómo podría resistir la práctica de la dramaturgia a tales operaciones?, y, si no puede resistir, ¿no debería ser descartada? (Heathfried, 2011: 93).

Es interesante observar que absolutamente todos los dramaturgos incluidos en este proyecto proceden de una implicación activa en el mundo del teatro: fundamentalmente son actores. Algunos comparten a la vez interpretación con dirección. Y algunos, incluso, tienen su propia compañía en la que participan como actores o directores. Este detalle es importante a la hora de considerar el daño que causa en ellos un desmantelamiento que supone, en definitiva, impedir el desarrollo de una vocación que habían derivado hacia lo profesional. Una de las preguntas a este respecto formuladas en el cuestionario a los autores era la de si preferían que sus obras fueran estrenadas o publicadas; a lo que respondió la totalidad que preferían ser estrenados. La respuesta de uno de ellos, José Luis de Blas, fue bastante contundente: "Estrenar es casi un mandamiento, una obra que no se estrena es una obra muerta" (De Blas, 2015: s. p.). Establece, además, este autor una personal proporción entre textos propios escritos frente a estrenados de cinco a uno. Como ya se ha destacado, casi todos estos autores han escrito para compañías independientes de Sevilla o han creado su propia compañía de teatro con la que han llegado a estrenar sus textos. Así, por ejemplo, Javier Berger está muy relacionado en sus estrenos con Los Ulen y Os Reverendos; José Luis de Blas ha montado varias compañías para estrenar textos concretos propios, pero posteriormente al estreno no han tenido continuidad; Jorge Dubarry, por su parte, mantiene una compañía propia que lleva por nombre ¿Por qué Teatro?, desde el año 1998; y hace dos años fundó, junto con otra de las actrices y dramaturgas incluidas en el proyecto de Lo Mínimo, María José Castañeda, la compañía Teatro del Gallinero, nacida tras varias creaciones en la primera temporada de Teatro Mínimo y con la que tratan, en palabras del autor, "temas actuales con carácter político-social bastante comprometidos" (Dubarry, 2015: s. p.).

En cuanto a la publicación de sus textos, estos autores manifiestan visiones similares que pasan en muchos casos por la autoedición. A las preguntas "¿Con qué dificultades te encuentras a la hora de publicar tu obra? ¿Cuántos textos tienes escritos y cuántos publicados?”, responden: 
Sólo tengo publicados 9 textos breves por mi cuenta. El único "problema" es aprender las herramientas para la edición profesional y el maquetado, pero como el tema me gusta... Luego, realmente imprimirlo no es tan caro. El ISBN cuesta 45 euros para un autor editor. Con la venta de pocos libros se puede amortizar rápido. Tener un libro es también una carta de presentación (De Blas, 2015: s. p.).

Ni hay lectores para el teatro, se ha perdido la tradición de la lectura teatral, apenas se enseña en los centros educativos... ;Llama al ministro de cultura y le comentas! Mis publicaciones vienen por tres vías, normalmente. 1.- La obra ha ganado un Concurso y dentro del premio se contempla la publicación. 2.- Alguna propuesta por parte de una Institución. 3.- La autoedición de una obra estrenada. / Tendré publicados una decena y escritos otros veinte (Berger, 2015b: s. p.).

\subsection{La crisis, la idea de libertad y el grotesco como bases conceptuales para analizar la nómina de obras de Teatro Mínimo}

La búsqueda fundamental de los cuarenta y tres autores que participaron en Lo Mínimo era el desarrollo de un teatro completamente libre, puesto que estaba al margen, como se ha comentado, de un basamento comercial. El libro electrónico final de la primera temporada es una selección de todas las piezas que se representaron. Dicha selección se hizo teniendo en cuenta las obras que tuvieron más afluencia de público. Los nueve autores y obras finalmente editadas fueron los siguientes: Culto a la abundancia, de Javier Berger; Edades, de José F. Ortuño; El valor del agua, de Joserra Leza; Emergencia Zombi, de Javier Berger; Gran Calibre, de Javier Berger; La zapatería de besugos, de Andrés Blanco; Mal en general, de David Montero; Parafilio o la breve historia del Sr. Maccowqueen y su amada Flora, de María José Castañeda; Políticamente Incorrecto, de Tomás Afán, Javier Berger y Paco Luna; Toda la verdad, de Jorge Dubarry y Joserra Leza.

Hay mucho de la mejor tradición bufonesca en el teatro de estos dramaturgos. Una mirada hacia la crítica grotesca y ácida de los bufones 
trasladada a los problemas sociales de la España del siglo XXI, que sin duda alguna se deja entrever en los diferentes argumentos, situaciones y personajes tipo que se plantean. Si se analiza el argumento de cada uno de los dramas, se observa la adscripción de los mismos a temáticas generales muy contemporáneas. Así, podemos destacar una primera línea que conviene denominar como Grupo de obras asociadas a la crisis, que constituye el núcleo más amplio de los intereses por argumentos y temáticas que se establecen en el libro, con un setenta por ciento de las obras recogidas. Dentro de este gran grupo cabría destacar, además, varios subgrupos definitorios del enfoque establecido en cada una de las obras concretas. Hallamos un primer subgrupo de obras que hablan abiertamente de la corrupción, analizada desde dos perspectivas distintas como son la política (robo y blanqueo de dinero por parte del político en el poder) y la especulación inmobiliaria (centrada en el ámbito de la destrucción del paisaje rural en pro de la construcción desaforada). Dos obras se asocian a este primer subtema de la corrupción: Toda la verdad ${ }^{8}$, sobre la corrupción política; y El valor del agua , en cuanto a especulación inmobiliaria. Otro subtema dentro de este Grupo de obras asociadas a la crisis sería el de la situación laboral, en el que se enfocan los argumentos desde la perspectiva del paro o el trabajo mal remunerado (lo que actualmente se denominan asalariados pobres), y que supondría un cuarenta por ciento de las obras recogidas en el libro. En este sentido encontraríamos las obras

\footnotetext{
${ }^{8}$ Toda la verdad, de Jorge Dubarry y Joserra Leza, es la confesión pública de un político corrupto antes de suicidarse, determinando así, desde él mismo, el establecimiento de una justicia para liberar su mala conciencia. Tiene un solo personaje. Esta obra fue estrenada en Teatro Mínimo Sevilla, librería Un gato en bicicleta, 2012. Dirigida e interpretada por Joserra Leza.

${ }^{9}$ El valor del agua, de Joserra Leza, expone diferentes situaciones enmarcadas en un ambiente rural; así habla de temas como la emigración, la inmigración (especialmente en cuanto a la llegada de ingleses y rumanos); y de la destrucción del campo a través de la especulación urbanística de los ayuntamientos en algunos pueblos. Tiene dos personajes. Esta obra fue estrenada en Teatro Mínimo Sevilla, librería Un gato en bicicleta. Estuvo representándose durante el mes de diciembre de 2012. Dirigida por Joserra Leza e interpretada por Belén Cobos, en el personaje de Francisca; y por Juan Ribadeneira, en el personaje de Jacinto.
} 
Mal en genera ${ }^{10}$, La zapatería de besugos ${ }^{11}$, Culto a la abundancia ${ }^{12} \mathrm{y}$ Políticamente incorrecto ${ }^{13}$. Sin salir de este gran grupo dedicado a la crisis, encontraríamos un nuevo subgrupo orientado hacia los recortes aplicados por el gobierno de Mariano Rajoy durante su primera legislatura, entre los años 2011-2015: concretamente nos estaríamos refiriendo a una obra, Emergencia Zombi ${ }^{14}$, centrada en uno de los recortes que más impacto social generaron en la población, como fue el que se llevó a cabo en la Sanidad

\begin{abstract}
${ }^{10}$ Mal en general, de David Montero, cuenta la historia de un personaje anodino, que fracasa uno tras otro en todos los trabajos de vendedor que le encarga su jefe, hasta que se ve probando suerte en su último trabajo que supone también su última oportunidad: el jefe lo ha enviado al Teatro Mínimo como intérprete. Tiene dos personajes. Esta obra fue estrenada en La seta coqueta, Sevilla. Dirigida por David Montero e interpretada por Antonio Estrada, en el personaje de Fulanito; y por el mismo Antonio Estrada, en el personaje de El Jefe, convirtiendo así esta obra en un unipersonal.

${ }^{11}$ La zapatería de besugos, de Andrés Blanco, sitúa a los personajes en una extraña y absurda conversación de besugos cargada de dobles sentidos, en torno al establecimiento de un contrato laboral que resulta imposible de llevar a cabo por parte empresario, a causa de los bajos ingresos de su zapatería. Tiene dos personajes. Esta obra fue estrenada en Teatro Mínimo Sevilla, librería Un gato en bicicleta, el 28 de febrero de 2013. Dirigida por Andrés Blanco e interpretada por el propio Andrés Blanco, en el personaje de Él; y por Betriz Cotobal, en el personaje de Ella.

${ }^{12}$ Culto a la abundancia, de Javier Berger, es una disparatada picaresca en la que se expone la historia de un falso pastor que insta a un grupo de feligreses a que le entreguen todo su dinero en nombre de Dios. Al final se descubre que el falso pastor es una mujer acuciada por una deuda de noventa mil euros de hipoteca que no sabe cómo pagar. Tiene dos personajes. Esta obra fue estrenada en noviembre de 2012, en la Sala El Cachorro, Sevilla. Dirigida por Jorge Dubarry e interpretada por María José Castañeda, en el personaje del Pastor; y por Jorge Dubarry, en el personaje del Ayudante.

${ }^{13}$ Políticamente incorrecto, de Tomás Afán, Javier Berger y Paco Luna acerca al espectador a un debate en torno a lo que es posible decir y lo que nunca estaría permitido, para obtener una subvención. Se plantean la capacidad de la sociedad y las instituciones para soportar formas de decir siempre provocadoras pero, ante todo, en completa libertad. Tiene dos personajes. Esta obra fue estrenada en Teatro Mínimo Sevilla, librería Un gato en bicicleta. Dirigida por Javier Berger e interpretada por Paco Luna, en el personaje de Frankie; y por el propio Javier Berger, en el personaje de Xabier.

${ }^{14}$ Emergencia zombi, de Javier Berger, traslada la acción a una España de futuro inmediato (año 2020) en el que el poder político ha decidido convertir a los yonkis en zombis para que se coman a los enfermos y así tener la capacidad de ahorrar gastos en el sistema de salud. Tiene dos personajes. Esta obra fue estrenada en Teatro Mínimo Sevilla, librería Un gato en bicicleta. Dirigida por Javier Berger e interpretada por el propio Javier Berger, en el papel de Bedel; y por Paco Luna, en el papel de Médico.
\end{abstract}


Pública. En un segundo gran grupo hallaríamos dos obras relacionadas con la Perversión sexual: la primera de ellas lleva por título Parafilia o la breve historia del señor McCowQueen y su amada Flora ${ }^{15}$, que, como se observa desde la primera palabra del título, narra la historia del amor de un granjero con varios de los animales de su granja; la segunda obra de este gran grupo lleva por título Gran calibre ${ }^{16}$, centrada en el tema de la perversión sexual dentro del clero. Por último, destacaríamos todavía un tercer gran grupo dedicado a la Incomunicación postmoderna y constituido en realidad por una única obra, Edades ${ }^{17}$, en la que nos adentramos en la intimidad psicológica de dos hermanas a lo largo de toda una vida, dentro de una sociedad contemporánea que incapacita a los seres humanos para la comunicación o bien la reduce al absurdo. En el siguiente esquema se facilita una visualización comprehensiva del análisis temático de las obras insertas en el libro:

${ }^{15}$ Parafilia o la breve historia del Sr. Maccowqueen y su amada Flora, de María José Castañeda, es definido por su propia autora como un "breve relato dramatizado utilizando el teatro de objetos". Asistimos a una historia de infidelidad amatoria entre un hombre y una vaca. Tiene dos personajes humanos, uno animal y uno vegetal, que son movidos por una sola actriz. Esta obra fue estrenada en Teatro Mínimo Sevilla, librería Un gato en bicicleta. Dirigida e interpretada por Eva Gallego, en la narración y las voces de todos los personajes.

${ }^{16}$ Gran calibre, de Javier Berger, presenta un cura que, mientras espera que lleguen los niños a la catequesis, ha contratado a un puto que resulta ser un completo inexperto en la materia, dado que es un padre de familia en paro que intenta ganar dinero como puede haciendo cualquier cosa. Ante la inexperiencia amatoria del puto, el cura mantiene una conversación con él, instruyéndolo acerca de técnicas masoquistas compartidas y en soledad. Tiene dos personajes. Esta obra fue estrenada en la Abacería Reinas, Sevilla. Dirigida por Javier Berger e interpretada por Miguel López, en el personaje de 1; y por Paco Luna, en el personaje de 2.

${ }^{17}$ Edades, de José F. Ortuño, es una exploración psicológica de dos hermanas a lo largo de distintas etapas de su vida, desde la adolescencia hasta la senectud, incidiendo en la incapacidad de comunicarse que se produce entre ellas. Tiene dos personajes. Esta obra fue estrenada en Teatro Mínimo Sevilla, librería Un gato en bicicleta. Dirigida por Laura Alvea e interpretada por Silvia Rey, en el personaje de La Una; y por Mercedes Bernal, en el personaje de La otra. Hubo además un estreno paralelo en Madrid, en la sala de Lucroteatro por dinero, con el mismo equipo, a excepción del personaje de La otra, que fue interpretado por Paqui Montoya. 
A. Grupo de obras asociadas a la crisis

A.1. Corrupción

A.1.1. Política: Toda la verdad.

A.1.2. Especulación inmobiliaria: El valor del agua.

A.2. Situación laboral: Mal en general; La zapatería de besugos; Culto a la abundancia; Politicamente incorrecto.

A.3. Recortes: Emergencia zombi.

B. Perversión sexual: Parafilia o la breve historia del señor McCowQueen y su amada Flora; Gran calibre.

C. Incomunicación postmoderna: Edades.

El absurdo, la ironía, lo escabroso y lo cínico podrían utilizarse como herramientas conceptuales para definir la generalidad de los mundos fabricados por estos autores y exhibidos a lo largo de la primera temporada de Teatro Mínimo. Si se analizan los argumentos expuestos en diversas notas al pie, se podrá percibir cómo cada una de estas herramientas de acercamiento teórico a la exposición temática aparecen con diferentes contingencias en todas estas obras. Si bien en unas se enfatizan más unos elementos que en otras. Así, las piezas comprendidas dentro del primer gran grupo señalado, el Grupo de obras asociadas a la crisis, desarrollan por encima de las demás los elementos del absurdo y la de la ironía. El elemento de lo absurdo está especialmente presente en los subgrupos segundo y tercero, es decir, el dedicado a la Situación laboral y el dedicado a los Recortes. La ironía, por su parte, es la herramienta que garantiza una crítica más completa en el subgrupo dedicado a la Corrupción. El conjunto de obras comprendido bajo el grupo denominado de la Perversión sexual, se centran fundamentalmente en lo escabroso, lo sórdido. A partir de estos elementos desarrollan una decadencia ambiental que sirve a los autores en el desarrollo psicológico de sus personajes. Y en tercer lugar, la obra comprendida bajo el epígrafe de la Incomunicación Postmoderna estaría regida por el elemento de lo cínico, como herramienta más apropiada para traducir la destrucción del factor humano en la sociedad actual, contemplada desde la incapacidad de sentir sorpresa emocional ante quien se tiene enfrente a lo largo de toda una vida. 


\section{CONCLUSIONES}

Retomando la pregunta inicial formulada desde el título del presente artículo, es necesario destacar a modo de conclusión ( $\mathrm{y}$ a tenor del desarrollo argumental expuesto), cómo el Teatro Mínimo comprende un primer momento de necesidad que evoluciona hacia un segundo momento de gusto en esa forma, especialmente detectado en la visión de libertad y experimentación que todos los autores persiguen en el mismo. Esto les lleva a ser especialmente críticos con el programa de subvenciones por parte de una institución pública como la Junta de Andalucía, pasando esta realidad de ser una mera crítica a la institución, a determinar un espacio de creatividad en el cual a lo largo de los años se han instalado y han aprendido a moverse extrayendo del mismo la independencia y la profunda libertad de expresión como una necesidad técnica para su construcción dramática. Por otro lado, la evidencia de que el setenta por ciento de las obras recogidas en el libro editado por la Junta de Andalucía permite observar cómo el Teatro Mínimo ha supuesto la garantía de una respuesta inmediata a la realidad política del país, imprime a estos autores una capacidad de reacción dramática casi periodística ante los acontecimientos sociales. Y todo ello a pesar de que los recortes han afectado a la institución pública y a partir de ahí se ha generado gran parte de la debacle que ha mermado gravemente el sector teatral en la comunidad andaluza. Hay que tener en cuenta que diversas publicaciones y el eco cultural por parte de la Junta de Andalucía, especialmente a través del CDAEA (Centro de Documentación de las Artes Escénicas de Andalucía), implica que siguen existiendo ciertas subvenciones $\mathrm{y}$, sobre todo, interés por la producción dramática en esta comunidad, lo que permite a los autores que se continúen difundiendo algunos de sus formatos más originales.

Desde un punto de vista exclusivamente empírico, es cierto que el proyecto de Teatro Mínimo fue decayendo poco a poco con el paso de los años. El futuro que pudiera haber tenido esta forma, quizás se haya mimetizado con el producto ofrecido desde el Microteatro. En este sentido habría que admitir que necesidad de forma y forma de necesidad se convirtieron en dos líneas estratégicas ligadas íntimamente a la circunstancia histórica que, especialmente en los primeros años del gobierno presidido por Mariano Rajoy, sucedió en Andalucía. Habría que plantearse, pues, que el éxito de este proyecto estaba muy ligado a la 
crítica social que desde diferentes sectores intelectuales generó un clima de necesario compromiso en los diversos ámbitos artísticos. Aunque más que morir, quizás el proyecto continúa latente en sus autores, esperando a ser reactivado cuando circunstancias similares de extrema necesidad requieran del teatro una respuesta directa, contundente y hasta pragmática a los interrogantes e inseguridades que pueda plantear el devenir social.

\section{REFERENCIAS BIBLIOGRÁFICAS}

BAJO MARTÍNEZ, M. J. (1996). "Panorama teatral de medio siglo de teatro en Andalucía". ADE Teatro 54-55, 28-34.

BARBA, E. (1997). Teatro. Soledad, oficio y revuelta. Argentina: Catálogos.

BERGER, J. (2015a). http://ringdelamentira.blogspot.com.es/ [15/10/2015].

(2015b). "Entrevista con Javier Berger". Inédita.

BERGER, J. y otros (2015). Lo mínimo: primera temporada Teatro Mínimo en Sevilla. Sevilla: Junta de Andalucía / Consejería de Cultura.

DE BLAS, J. L. (2015). "Entrevista con José Luis de Blas". Inédita.

DUBARRY, J. (2015). "Entrevista con Jorge Dubarry". Inédita.

ESPEJO ROMERO, R. (2005). "Un paseo por el teatro breve andaluz contemporáneo". En Teatro breve andaluz, Ramón Espejo Romero (ed.), 5-29. Sevilla: Junta de Andalucía.

GUTIÉRREZ CARBAJO, F. (ed.) (2013). Teatro breve actual. Barcelona: Castalia

HEATHFRIED, A. (2011). "Dramaturgia sin dramaturgo". En Repensar la dramaturgia. Errancia y transformación, VV. AA, 91-103. Murcia: CENDEAC

MAYORGA, J. (2001). Teatro para minutos. Ciudad Real: Ñaque.

MICROTEATRO EN MADRID (s. f.). http://microteatromadrid.es/ [10/11/2015].

PAVIS, P. (1998). Diccionario del teatro. Dramaturgia, estética, semiología. Barcelona: Paidós.

PÉREZ-RASILLA, E. (2008). "Jardiel Poncela y otros autores". En Historia del teatro breve en España, Javier Huerta Calvo (dir.), 1153-1161. Madrid: Iberoamericana Vervuert. 
QUINTANA, R.(2008). Una vida de comediante. La aventura interminable. Sevilla: Suranda.

ROMERA CASTILLO, J. (ed.) (2015). El teatro breve en los inicios del siglo XXI. Madrid: Visor Libros.

ZURRO, A. (2010). "Presentación". En Día mundial del teatro, Sevilla, [1-4]. Sevilla: Junta de Andalucía. (2006). "Prólogo". En 60 obras de 1 minuto de 60 autores dramáticos andaluces, [1-4]. Sevilla: Junta de Andalucía.

Recibido el 8 de enero de 2019.

Aceptado el 11 de abril de 2019. 